\title{
Computer Assisted Learning: An Application to Corporate Failure Prediction Models and Analysis
}

http://dx.doi.org/10.3991/ijes.v2i1.3681

A. C. H. Marques, Federal University of São Carlos - UFSCar, São Carlos-SP, Brasil.

Abstract - This paper seeks to show the relation between learning complex problems and facilitation of application software. From the concept of computer assisted learning, the problem of failure of companies in administration was picked. Initially, the model was made by analytical-numerical methods only with applied mathematics tools and then with the aid of computational tools. Learning was measured by an exponential learning curve.

Index Terms - analytical-numerical methods, computer assisted learning, learning curve, model of unsuccessful of companies.

\section{Aprendizagem Assistida por Computador: Uma Aplicação ao Modelo de Análise de Insucesso de Empresas}

\author{
A. C. H. Marques \\ Universidade Federal de São Carlos - UFSCar/DiFO, São Carlos-SP, Brasil
}

\begin{abstract}
Resumo-Este trabalho procura mostrar a relação entre a aprendizagem de problemas complexos com a facilitação de softwares de aplicação. A partir do conceito de aprendizagem assistida por computador, escolheu-se o problema de insucesso de empresas na área de administração. Inicialmente a modelagem foi feita por meio de métodos analítico-numéricos apenas com as ferramentas da matemática aplicada e depois com o auxílio de ferramentas computacionais. A aprendizagem foi medida através da curva de aprendizagem exponencial.
\end{abstract}

Palavras-chave-aprendizagem assistida por computador, curva de aprendizagem, métodos analítico-numéricos, modelo de insucesso de empresas.

\section{INTRODUÇÃO}

Com a popularização do acesso ao computador e considerando-se o contínuo aumento da capacidade de armazenamento de dados, velocidade de processamento e recursos das técnicas de programação, passou-se a desenvolver abordagens computacionais que melhor assistam ao raciocínio humano, fazendo com que a máquina transforme-se em um novo recurso para dinamizar a interface homemmáquina, fazendo com que a informação possa ser mais rapidamente trabalhada e assimilada com o auxílio do computador.

Dentro de um panorama geral das abordagens para sistemas computacionais nomeia-se, conforme [1], "ensino assistido por computador" à classe de sistemas que exemplificam o paradigma instrucionista de aprendizagem e detêm o controle da interação; "ambientes interativos de aprendizagem", à classe de sistemas que exemplificam o paradigma construcionista e cujo controle da interação está totalmente nas mãos do aprendiz ou é compartilhado entre o aprendiz e o sistema; e "aprendizado socialmente distribuído", á classe que representa as novas possibilidades surgidas com a Internet e a globalização da informação.

Nesse cenário, este artigo apresenta o uso desses recursos para uma abordagem sistemática de tratamento e análise unificada, com características interativas, e que permite, simultaneamente, a aprendizagem de conceitos teóricos, manipulação numérico-analítica de equações matemáticas, técnicas de solução e análise fenomenológica, por meio de recursos gráficos e animação.

A figura 1 ilustra a sistematização básica das ferramentas e formalismos utilizados para promover a abordagem do aprendizado assistido por computador, a partir do tratamento unificado das áreas de conhecimento, [2].

Do ponto de vista educacional, as práticas pedagógicas atuais levam em conta novo paradigma educacional que é a tecnologia novas possibilidades metodológicas. A tecnologia altera o processo de interesse no processo educativo transformando a aprendizagem interessante para os educandos e o computador como mecanismo virtual de aprendizagem para o melhoria da leitura, interpretação e desenvolvimento visual e criativo.

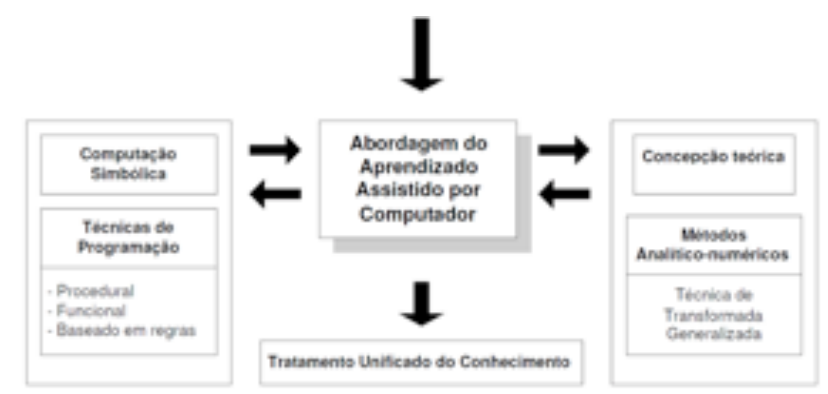

Figura 1. Abordagem unificada do conhecimento 
Grande parte dos estudos que tratam da aprendizagem voltam-se para a educação nos anos fundamentais e poucos buscam mensurar as dificuldades da aprendizagem conteudista do aluno na universidade. Autores como [3] mostram que, para entender o conhecimento, é preciso levar em conta a organização dos conhecimentos que o sujeito cognoscente realiza nas situações concretas. Compreender a construção do conhecimento depende não só de observar o processo, como também de transformá-lo. É recorrente nas áreas de exatas a busca por privilegiar o processo de construção de conceitos, sem levar em conta as dificuldades reais vividas pelos alunos ou impostas pelos próprios conteúdos, bem como suas relações com o processo didático de abordagem do conceito.

A partir desse referencial teórico, procurou-se mensurar a aprendizagem através da abordagem de um problema que não fosse estritamente afeito à linguagem simbólica físico-matemática (notadamente complexa), mas um problema que envolvesse uma realidade prática com certa complexidade de modelagem e que exigisse reflexão quanto ao processo decisório. A área de administração financeira de empresas é rica nesse tipo de problemas, mais especificamente gerenciamento financeiro.

Tomando-se o caso de análise gerencial financeira assume-se implicitamente que uma empresa pode durar indefinidamente enquanto funcionar em condições normais. Isto é uma generalização e deve-se analisar a teoria em uma situação de insolvência técnica, ou seja, a impossibilidade da empresa cumprir seus compromissos no curto prazo. A teoria financeira aponta vários estudos sobre insolvência, centrados principalmente na busca de variáveis que ajudem a predizê-la. Tomando-se como base os estudos de [4], [5], [6] e [7] nota-se a forte predominância dos enfoques de falência através de certas razões financeiras e, fazendo uso da técnica de análise discriminante, pode-se predizer, com certa antecipação e algum grau de confiabilidade, a falência de uma empresa.

O modelo de insucesso, tomado como exemplo neste trabalho, considera como básico o conceito "fluxo de caixa" e procura evidenciar as variáveis incluídas no fluxo de caixa que o gerente deverá administrar. Conforme [7], o fluxo de caixa para o modelo será definido em função da margem de lucros, rotação de ativos e nível de endividamento. Em outras palavras, considera-se que o fluxo de caixa não é uma série de dados ex-post ou ex-ante, mas sim o resultado de decisões tomadas em aspectos implícitos nas variáveis margem de lucro, rotação e endividamento, tais como preços, custos, canais de venda, combinação de produtos, níveis de inversão, entre outros. Esse modelo tem um corpo teórico próprio e conceitos que são definidos para seu propósito, portanto válidos somente dentro do esquema teórico proposto.

A proposta deste trabalho é mostrar, através de uma curva de aprendizagem, a aplicação da aprendizagem assistida por computador em um problema complexo envolvendo a concepção do modelo (compreensão de um problema da administração gerencial); modelagem simbólica matemática; equacionamento seguido das hipóteses e restrições; e uma generalização do modelo.

O caráter dinâmico de acesso aos estágios do processo do aprendizado, sob a ótica do tratamento unificado do conhecimento, fica reforçado pelos recursos de programação existentes, por exemplo, no software "Mathematica"
${ }^{1}$, que foi o adotado neste trabalho por apresentar recursos adicionais, nos estados associados à interpretação fenomenológica, tabelas, gráficos e recursos de animação gráfica, disponibilizados ao longo da navegação no ambiente de aprendizagem.

Em todas as fases, a utilização do software será apontada e o desempenho obtido para solução do problema será computado para a comparação de duas curvas médias de aprendizagem: a curva de aprendizagem sem os recursos de aprendizagem assistida por computador e a curva de aprendizagem com os recursos de aprendizagem assistida por computador.

\section{A. Modelo margem-rotação-endividamento (MRE)}

Define-se condição de insucesso de uma empresa a possibilidade do não-pagamento dos compromissos financeiros no curto prazo. Isto não implica em falência, que se refere ao processo judicial de fechamento de empresa. Desse modo, o insucesso não necessariamente implica chegar à quebra judicial, já que antes da falência podemse obter arranjos voluntários entre credores e devedores através da moratória das dívidas, capitalização das dívidas, liquidação de alguns ativos de decretação de continuidade de giro, entre outras.

O insucesso, em termos operativos, pode ser expresso como a não-cobertura dos compromissos financeiros contratuais com os fluxos de caixa esperados. Esse processo implica que a empresa, ao seguir operando em situação de não cobertura de seus compromissos com o valor presente de seus fluxos de caixa, pode levar a diminuição do patrimônio dos proprietários. Tem efeito econômico-financeiro de dupla implicação, pois o insucesso (problema financeiro) pode conduzir à diminuição do patrimônio (problema econômico). Daí a denominação de insucesso econômicofinanceiro a este processo. A origem do insucesso econômico-financeiro deve-se geralmente a uma má gestão empresarial, a qual pode conduzir uma empresa, por motivos internos ou externos, a uma posição crítica de fechamento. Os sintomas do insucesso podem se observados através de diferentes indicadores financeiros tradicionais de solvência e liquidez. O modelo analítico pretende explicar o insucesso tomando como variáveis-chave a margem de lucros, a rotação de ativos e o endividamento.

Conforme [5], o modelo margem-rotaçãoendividamento consiste em decompor diferencialmente a rentabilidade dos investidores em seus elementos básicos, a saber: margem, rotação e endividamento, analisando o peso relativo de cada uma destas variáveis e sua influência na rentabilidade. Para isso, calculam-se as produtividades marginais da margem de lucro, da rotação e do endividamento que medem o peso relativo de cada componente. Trata-se, portanto, de um modelo linear que, na forma empírica, obtém-se usando as técnicas de regressão. A expressão geral é dada por:

$$
d R=\beta_{1} d m+\beta_{2} d r+\beta_{3} d e
$$

onde $d R$ é variação da rentabilidade dos investidores de um período em relação a outro; $d m$ é variação da margem de lucros de um período em relação a outro; $d r$ é a variação de rotação (vendas sobre Ativo) de um período em relação a outro; de é a variação do endividamento (Ativo

1 É um sistema computacional usado em muitos campos científicos, engenharia, matemática e algumas áreas da computação. Foi concebido por Stephen Wolfram e é desenvolvido pela Wolfram Research - Champaign, Illinois. 
sobre Capital) de um período em relação a outro; $\beta_{1}, \beta_{2}$ e $\beta_{3}$ produtividade marginal de margem de lucro, da rotação e do endividamento, respectivamente. As produtividades marginais são variáveis exógenas para o modelo e $d m$, $d r, d r$ são as variáveis endógenas.

Esse modelo estabelece estratégias a serem seguidas para cada empresa [5] e os riscos econômicos de liquidez de endividamento são medidos pela variância das variações da margem de lucro, da rotação e do endividamento, respectivamente $^{2}$. O risco total de rentabilidade é definido como a variância das variações da rentabilidade ${ }^{3}$.

\section{A.1 Hipóteses do modelo}

- Existe comportamento racional econômico, ou seja, que os empresários maximizam seu patrimônio, expresso como o valor presente dos fluxos esperados de caixa.

- É possível determinar estatisticamente as produtividades marginais da margem, da rotação e do endividamento.

- Os conceitos utilizados têm validade dentro do contexto do modelo margem-rotaçãoendividamento, [7].

- Existem custos falenciais.

- A fonte principal de recursos para cobrir os compromissos financeiros (pagamento de juros de passivos e amortizações de capital) são os fluxos operacionais.

- $\quad \mathrm{Na}$ função do valor presente dos fluxos de caixa trabalha-se com capitalização de juros na forma contínua.

O modelo pode ser utilizado para simular a gestão do próximo período a partir do postulado de que se deve maximar o valor presente dos fluxos de caixa esperados, sujeitos a certas restrições econômico-financeiras. Pretende-se, desta forma, maximar a seguinte função:

$$
V=\int_{0}^{n} F_{t} e^{k t} d t
$$

Onde $V$ é valor do patrimônio da empresa; $F_{t}$ o fluxo de caixa esperado no período $t$ e $K$ o custo de Capital (taxa de juros) exigida pelos acionistas. Se $n$ for muito grande e for igual para todos os períodos, então (2) transforma-se em:

$$
V=\frac{F_{t}}{K}
$$

Pondo $V$ em função das variáveis $\$ d m, d r$ e $d e$, tem-se que a função a maximizar é:

$$
V(d m, d r, d e)=\frac{(d m+1)(d r+1)(d e+1) R_{t-1}}{K}
$$

\footnotetext{
2 Define-se variância das variações da variável $d a$ $a: \sum(d a-\bar{d} a)^{2} /(n-1)$, onde $d a$ pode ser igual à margem, à rotação ou ao endividamento, segundo o caso.

3 Não se fará uma discussão teórica acerca do uso da variância como medida do risco
}

onde $d m$ variação da margem de lucros entre os períodos $t$ e $(t-1) ; d r$ variação de rotação de ativos entre os períodos $t$ e $(t-1) ; d e$ variação do endividamento entre os períodos $t$ e

$(t-1)$ a rentabilidade dos proprietários no período

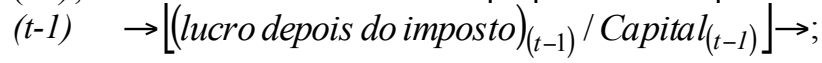
$C$, Capital no período $t$; e $K$ custo de Capital.

\section{A.2 Maximização do modelo}

$\mathrm{Na}$ equação (4), as variáveis endógenas são $d m, d r\} \boldsymbol{e} d e$ e o restante são variáveis exógenas. Esta função, ao ser maximizada, incrementa o valor do patrimônio e pode-se visualizar explicitamente a incidência das variáveis da gestão financeira - margem de lucro, rotação e endividamento. Além disso, observa-se que o valor da empresa em $t$ depende da rentabilidade obtida no período imediatamente anterior.

A função a maximizar tem restrições que condicionam sua otimização, relacionadas às ações que podem provocar insucesso, e que se traduzem em não cumprir os compromissos financeiros com o fluxo operacional. Portanto, a restrição, em termos operativos, consiste em igualar o fluxo operacional ao valor dos compromissos financeiros. Nesse caso, o compromisso financeiro é o pagamento de juros e amortizações de passivos.

Supondo que existam passivos iguais a $S P$, contratados a uma taxa de juros por $n$ períodos, então o pagamento por capital e juros por período é dado por $\frac{p_{i}}{1-e^{-i n_{1}}}=$ amortização + juros $; \quad e=2,7182$.

Dispõe-se de um fluxo $F t$ que é igual ao lucro depois de impostos $(U t)$ mais o valor da depreciação dos ativos $(D t)$. Nesse caso, supõe-se que não existam outros lançamentos contábeis que afetem o lucro, tais como as amortizações do Ativo nominal e ajustes por inflação. Mantendo-se a restrição de compromissos financeiros, deve-se verificar que o fluxo operacional deve ser igual aos compromissos financeiros de tal forma que assegure o funcionamento da empresa no longo prazo, o que, expresso em termos matemáticos, será:

$$
U+D=\frac{p_{i}}{1-e^{-i n_{2}}}
$$

Se o Ativo $A$ deprecia-se em $n$ períodos, a uma taxa $d$, dividindo-se a igualdade (5) pelo Capital e rearranjando os termos, tem-se:

$$
\frac{U}{C}=\frac{P}{C}\left(\frac{i}{1-e^{-i n_{1}}}\right)-\left(\frac{A}{C}\right)\left(\frac{d}{1-e^{-i n_{2}}}\right)
$$

Se considerarmos que tanto a empresa como os ativos têm vida longa, então (6) é convertida em:

$$
\frac{U}{C}=\frac{P}{C}(i)-\frac{A}{C}(d)
$$


A relação de endividamento é igual a $\frac{i+P}{C}=\frac{A}{C} e$, sabese que $\frac{U}{C}=(e-1) i+d e, \frac{U}{C}=R_{t}$,

$R_{t}=R_{(t-1)} *(d R+1) \rightarrow$ rentabilidade do capital em $t$ $m_{t}=m_{(t-1)} *(d m+1) \rightarrow$ margem de lucros em $t$; $r_{t}=R_{(t-1)} *(d r+1) \rightarrow$ rotação de ativos em $t$; $e_{t}=e_{(t-1)} *(d e+1) \rightarrow$ relação de endividamento em $t$ então, rearranjando os termos e substituindo em (7), temse:

$$
d R=\frac{e_{(t-1)}}{R_{(t-1)}}(d e+1)(1+d)-R_{(t-1)}
$$

Substituindo (1) em (8) e rearranjando, tem-se:

$$
\begin{aligned}
H(d m, d r, d e)= & \beta_{1} d m+\beta_{2} d r+d e\left[\beta_{3}-\frac{e_{(t-1)}}{R_{(t-1)}}\right] \\
& ((i+d)-)-\frac{e_{(t-1)}}{R_{(t-1)}}(i+d) \frac{i}{R_{(t-1)}}=0
\end{aligned}
$$

A igualdade dada por (9) é a restrição do modelo e seu significado é a garantia de que o fluxo operacional do negócio permitirá cobrir o pagamento dos compromissos por passivos, isto é, a quota de amortização do capital de juros que este gera, é, de fato, uma das condições que impedem o insucesso econômico-financeiro Deve-se assinalar que essa restrição não assegura o pagamento dos dividendos aos proprietários por uma razão operacional, já que, perto da insolvência técnica, é possível que os proprietários não peçam distribuição de lucros, embora no longo prazo deva-se incorporar ao lado direito de (8) o valor dos dividendos como restrição de insucesso e que, ao cumprirse, permitirá assegurar o funcionamento normal da empresa.

Assim, a primeira etapa do modelo pode ser resumida a "maximizar o valor do patrimônio expresso na igualdade dada por (4) sujeito à restrição de igualdade dada por (8) ', ou seja, constrói-se a seguinte função langrangiana:

$$
U=(d m, d r, d e)=V_{t}+\lambda H_{t}
$$

de forma que,

$$
\begin{gathered}
\frac{\partial u}{\partial m}=(d r+1)(d e+1)\left(\frac{R_{(t-1)}}{K}\right) C_{t}+\lambda \beta_{1}=0 \\
\frac{\partial u}{\partial r}=(d m+1)(d e+1)\left(\frac{R_{(t-1)}}{K}\right) C_{t}+\lambda \beta_{2}=0 \\
\frac{\partial u}{\partial e}=(d m+1)(d r+1)\left(\frac{R_{(t-1)}}{K}\right) C_{t}+\lambda\left[\beta_{3}-\right] \\
\left.\left.\frac{\partial u}{\partial e}=\beta_{1} d m+\beta_{2} d r+d e\left[\beta_{3} \frac{e_{(t-1)}}{R_{(t-1)}}(i+K)\right]-\frac{e_{(t-1)}}{R_{(t-1)}}\right)(i+d)\right)
\end{gathered}
$$

$$
(1+d)-\left(\frac{i}{R_{(t-1)}}\right) R_{(t-1)}=0
$$

O significado das igualdades dadas por (11) a (13) implica em otimizar a função $U$, então, os valores da função aumentam diante de pequenas variações nas variáveis margem de lucro, rotação e endividamento. Assim, a igualdade dada por (11) mostra que qualquer incremento na margem $(\mathrm{dm})$ provoca uma modificação no lucro global $U$; analogamente, pode-se interpretar (12) e (13). A igualdade dada por (14) significa que se o fluxo operacional modifica-se em $\$ 1$, então, o lucro marginal modificar-se-á segundo a expressão do lado direito da igualdade, isto é, mede a variação infinitesimal no lucro devido a uma pequena variação no fluxo operacional. Assim, se o multiplicador de Lagrange , então, um aumento no fluxo operacional origina um incremento no lucro.

Como auxilio computacional para a aprendizagem dos multiplicadores de Lagrange pode-se usar uma aplicação bastante simples, porém esclarecedora conceitualmente: determinar as dimensões de uma caixa retangular, sem tampa, para um dado volume, de tal forma que o material usado na sua confecção tenha mínima quantidade. A solução deste problema, utilizando o software Mathematica [8], pode ser vista nas figuras 2 e 3 .

Entretanto, mais do que a solução algébrica do sistema de equações lagrangianas, o que nos interessa são as interpretações e deduções da política financeira que o sistema envolve. A solução do sistema de equações (10) a (14), resulta, para o ponto ótimo, as seguintes relações:

$$
\beta_{1}(d m+1)=\beta_{2}(d r+1)=(d e+1)\left[\beta_{3}-\left(\frac{e_{(t-1)}}{R_{(t-1)}}\right)(i+c\right.
$$

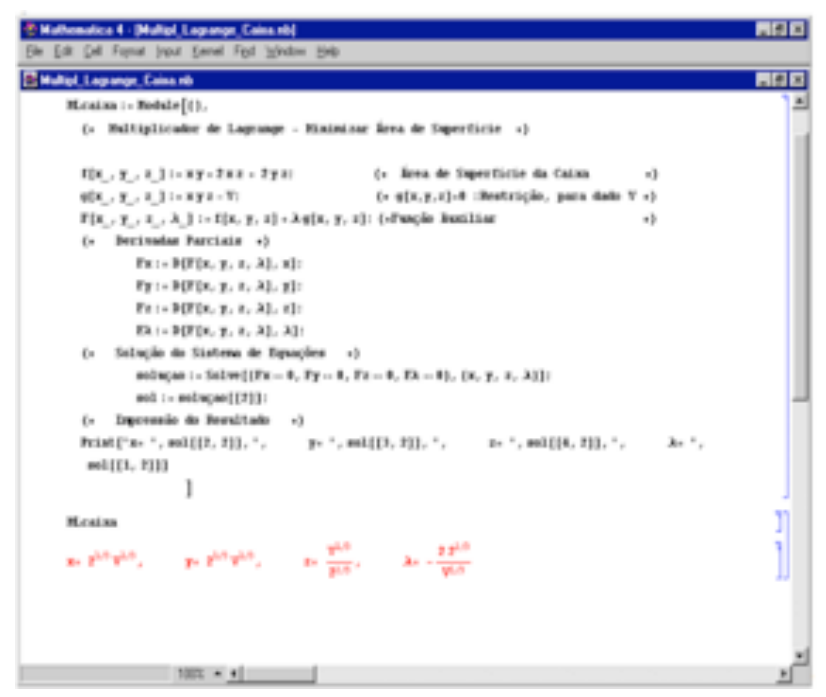

Figura 2. Solução dos multiplicadores de Lagrange - Mathematica. 


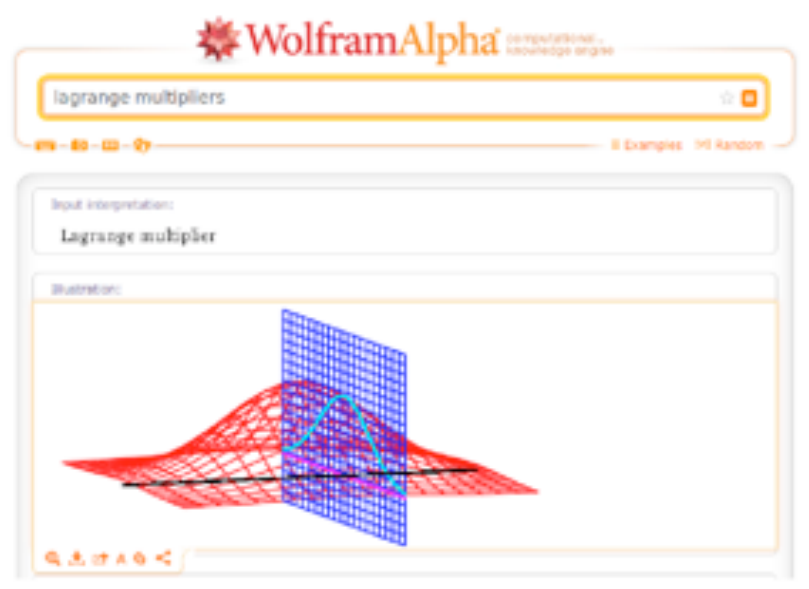

Figura 3. Multiplicadores de Lagrange - Mathematica online.

A igualdade (16) indica que no ponto ótimo (evita-se o insucesso financeiro) a variação total na margem deve ser igual à variação total na rotação. Do modelo MRE sabe-se que $\beta_{i} d_{i},(i)=$ margem, rotação, endividamento) é a variação total atribuída a cada uma das variáveis. A principal implicação da política financeira é que qualquer incremento que se espere na margem deve estar necessariamente relacionado ao incremento esperado na rotação e no endividamento. $\mathrm{O}$ modelo original de MRE não mostra níveis ótimos para as variáveis $d^{*} m, d^{*} r$ e $d^{*} e$, tal como indicado anteriormente. Dessa versão podem-se inferir as políticas de empresa de forma a avaliar o impacto que terão determinadas estratégias de preços, custos e financiamento na margem de lucros, na rotação e no endividamento.

Calculando-se os valores das variáveis $d m, d r$ e de no ponto ótimo, a partir do sistema de equações lagrangianas, tem-se ${ }^{4}$ :

$$
\begin{gathered}
d^{x} m=\frac{\beta_{2}+\beta_{3}-2 \beta_{1}-\left(\frac{i}{R_{(t-1)}}\right)+R_{(t-1)}}{3 \beta_{3}} \\
d^{x} r=\frac{\beta_{1}+\beta_{3}-2 \beta_{2}-\left(\frac{i}{R_{(t-1)}}\right)+R_{(t-1)}}{3 \beta_{2}} \\
d^{x} e=\frac{\beta_{1}+\beta_{2}-2 \beta_{3}-\left(\frac{i}{R_{(t-1)}}\right)+R_{(t-1)}(i+d)}{3 \beta_{1}}
\end{gathered}
$$

\section{A.3. Generalização do modelo}

$\mathrm{Na}$ análise do modelo desenvolvido há alguns aspectos que foram negligenciados, a restrição imposta é única e pode esse conjunto levar a uma explicação simplificada da realidade. Para ser mais realista, serão considerados dois aspectos que alteram operacionalmente a solução estabelecida (16) a (18).

4 Uma comprovação desta dedução pode ser obtida através do software Mathematica e também pode ser usada como exercício de aplicação de modelagem numérico-computacional.
Um primeiro aspecto é a estimação da restrição financeira imposta. Foi considerado um único ponto dessa restrição, já que supôs-se que os fluxos operacionais cobrem em sua totalidade o pagamento dos compromissos. Entretanto, deve-se esperar, para uma empresa que busque o crescimento, que os fluxos operacionais superem os compromissos de passivos de forma a poder cumprir obrigações com proprietários e pagar dividendos. Em outras palavras, a restrição $H(d m, d r, d e)$, expressa na igualdade (9), passa a ser uma desigualdade cujo limite inferior é zero. Esta nova condição faz mudar operacionalmente o método de otimização já apresentado.

Um segundo aspecto, para o caso de empresas que poderiam fracassar, é o custo implícito e explícito da insolvência, tais como:

custos de limitação, ou diminuição do valor dos ativos devido à venda a preços menores que aqueles quando a empresa não encontra-se em situação de insucesso financeiro (insolvência);

custos denominados "custos indiretos de insolvência", tais como redução das vendas pela menor credibilidade dos fornecedores; custos da perda de tempo dos executivos, devido a terem que se dedicar ao processo de insolvência; e altas súbitas nos custos de produção por liquidação de alguns ativos de produção;

gastos próprios do processo de insolvência por compensações a terceiros, tais como advogados, síndicos, contadores, leiloeiros, etc.;

perdas impositivas de poupanças futuras.

Para a análise de um modelo global de insucesso, os custos mais importantes são os relacionados ao menor valor que pode ter o conjunto dos ativos no momento da liquidação, ou seja que esses ativos possam ter um valor mais baixo que os passivos que se deve pagar. Supondo que os ativos caiam em $\$ q$ por cada $\$ 1$ em caso de insolvência, então deve-se verificar como condição básica

$$
A(1-q) P
$$

onde $A$ é o total de Ativos e $P$ é o total de Passivos.

Fazendo transformações algébricas, a desigualdade (19) transforma-se em:

$$
q e_{(t-1)} d e+q e_{(t-1)} 1
$$

A desigualdade (20) é uma restrição imposta ao modelo, sendo seu objetivo incluir o menor valor que pudessem ter os ativos, no caso em que o insucesso financeiro transforma-se em falência. Com a incorporação dessas condições, o problema reduz-se a determinar o valor máximo de um sistema de inequações e que pode ser resolvido usando-se as condições de Kuhn-Tucker, que originam o seguinte sistema de equações (condições de primeira ordem):

$$
\begin{gathered}
L=\varphi(d m, d r, d e)+\lambda_{1} H_{1}+\lambda_{2} H_{2} \\
\frac{\partial L}{\partial m}=\frac{(d r+1)(d e+1)}{K} C+\beta_{1} \lambda_{1} \leq 0 \\
\frac{\partial L}{\partial r}=\frac{(d r+1)(d e+1)}{K} C+\beta_{2} \lambda_{1} \leq 0
\end{gathered}
$$




$$
\begin{gathered}
L=\varphi(d m, d r, d e)+\lambda_{1} H_{1}+\lambda_{2} H_{2} \\
\frac{\partial L}{\partial e}=\frac{(d m+1)(d e+1)}{K} C+\left[\beta_{3}-\left(\frac{e_{(t-1)}}{R_{(t-1)}}\right)(i+d)\right] \lambda_{1} \\
-e_{t-1} q \lambda_{2} \leq 0
\end{gathered}
$$

Uma forma de verificação dessas equações pode ser obtida usando o software Mathematica, como mostrado na figura 4.

De acordo com o exposto anteriormente, se os custos de insolvência não são suficientemente importantes para impedir que os ativos líquidos sejam superiores aos passivos, então este modelo geral conduz ao modelo clássico MRE. Em termos matemáticos, isso implica em que se $q \leq \frac{i}{e_{t}}$, então $A(1-q) \geq C$, portanto, a segunda restrição, que condiciona o problema, não é obrigatória, isto é, diferente de zero, o que, pelo teorema de Kunh-Tucker, obriga o valor do lagrangiano $\lambda_{2}=0$, o que transforma o modelo generalizado no primeiro; ou seja, o primeiro modelo é um caso particular que pode adquirir validade quando a empresa tem ativos tais que possam servir para pagar os compromissos financeiros, apesar da existência dos custos de insolvência.
O propósito desses modelos é apresentar um marco teórico para o insucesso financeiro de uma empresa, de forma a oferecer ao administrador opções para decisões contingenciais e estratégicas sem perder de vistas as variáveischave que definem a boa administração de empresas. As soluções algébricas dos modelos produzem valores de variações da margem, da rotação e do endividamento, que orientarão as política e táticas financeiras. A dificuldade que se enfrenta, portanto, é determinar quais valores limites para essas variáveis evitarão possíveis insucessos. Nessa perspectiva, os insucessos de empresas seriam uma consequência, basicamente, de uma determinação incorreta das tendências futuras das variáveis macroeconômicas. Esses erros do presente são os que levam as empresas a dificuldades quando se deparam com futuros problemas socioeconômicos, isto é, o insucesso da empresa começa a originar-se antes das crises macroeconômicas ao não se avaliar corretamente as tendências básicas.

Ao se fazer a generalização do MRE, desenvolve-se uma abstração estabelecendo certos padrões, com coerência teórica, que permitiram relacionar fatos econômicos de insucesso de empresa, apesar de incorrer-se em simplificações à luz das hipóteses para a aplicabilidade, à qual depende da validade e confiabilidade na determinação das produtividades marginais de rotação, margem e endividamento.

nFe: $=$ Module $[(1),(* \quad \rightarrow)$

(* V é a funçio que desejanos nacinizax *)

$V\left[d n, d r, f v_{-}\right]:=((\mathrm{dn}-1)+(\mathrm{dr}-1)+(\mathrm{dr}-1), \mathrm{C}) / \mathrm{k}:$

(* $M[X, Y, z]=0$; Restriçló, dudo $\circ$ alR *)

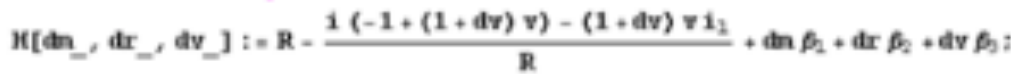

(* Funcäo Avociliar *)

U[n, $d r$, $\left.d v, \lambda_{-}\right] \leqslant V[d n, d r, d v]+\lambda, M[d n, d r, d v]:$

(A) Derivallas Parciais v)

Uan : - D [U[an, dr, $d v, x], d n]$;

Udr : = D [u[dn, $\mathrm{dr}, \mathrm{dr}, \lambda]$, dr ]:

Udv : = D [U [dn, $\mathbb{d r}, d r, \lambda], d v]$ :

ID : = D [U[dn, dr, dv, $\lambda], \lambda]$ :

(* Soluçáo do Sistema de Eruaçöes -)

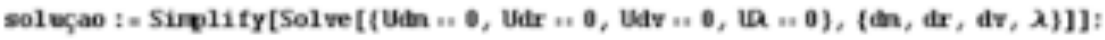

sol $\mathrm{i}$ soluçao $[$ [4]]:

(* Treressilo do Resultado *)

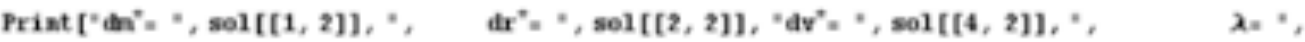

$\operatorname{sol}[[3,2]]] \quad]$

nes

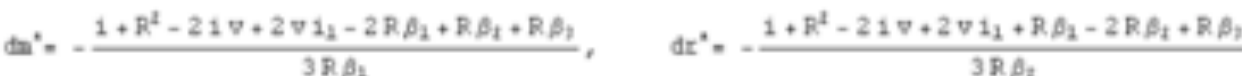

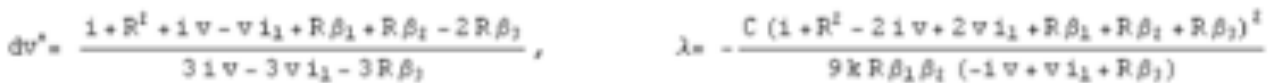

$1008+4$

Figura 4. Solução do sistema de inequações - Mathematica 


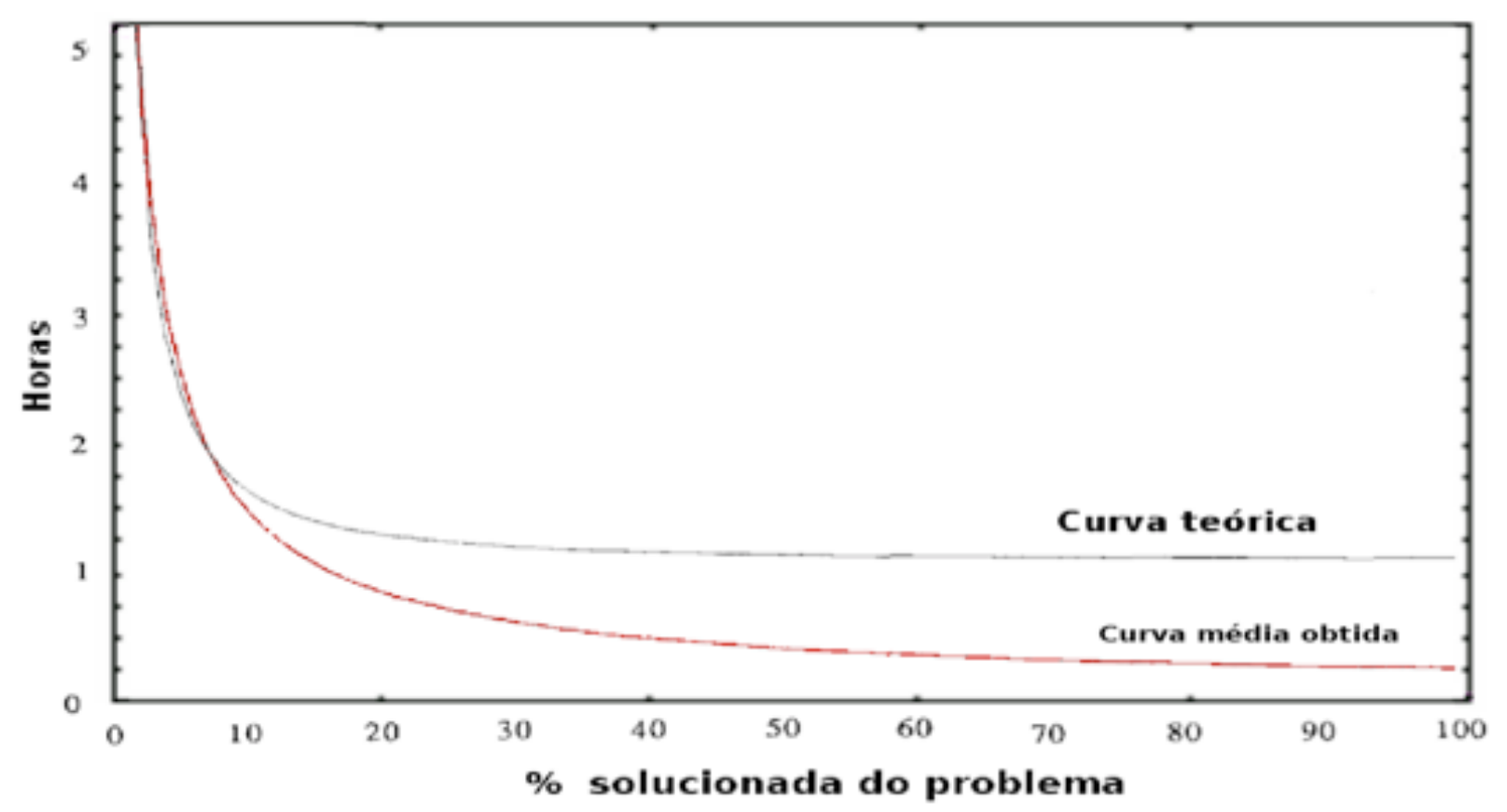

Figura 5. Comparativo entre a curva de aprendizagem teórica e a apurada.

O processo de determinação das produtividades marginais apresenta certas dificuldades que podem distorcer os resultados, como por exemplo: problemas de formulação do modelo MRE, multicolinearidade entre taxas, estimação do modelo global e existência de erros estatísticos.

\section{B. Curva de aprendizagem}

De acordo com [9], conhecimento é uma mistura fluida de experiência condensada, valores, informação contextual e insight experimentado, a qual proporciona uma estrutura para a avaliação e incorporação de novas experiências e informações com origem e aplicação na mente dos conhecedores. A aprendizagem para solução de problemas complexos requer um alto nível de liberdade para que o aprendiz apreenda a estrutura conceitual do problema. Depois de transformar o problema complexo em problema simples, é necessário um alto nível de segurança para operacionalizar as relações para tomada de decisão.

Uma forma bastante reconhecida no meio acadêmico, e aceita como método educativo no ensino superior, é conhecida como Aprendizagem Baseada em Problemas que estabelece uma estratégia pedagógica centrada no aluno, onde se procura que este aprenda por si próprio. $\mathrm{O}$ modelo é elementar: apresenta-se um caso para estudo aos estudantes; em seguida, os estudantes formam grupos de trabalho, identificam o problema, investigam, debatem, interpretam e produzem possíveis soluções ou resoluções, ou recomendações. De forma resumida, os alunos são confrontados com problemas contextualizados e pouco estruturados e para os quais empenham-se em encontrar soluções significativas.

Nas organizações, o conhecimento costuma estar embutido não só em documentos ou repositórios, mas também em rotinas, processos, práticas e normas organizacionais. $\mathrm{O}$ efeito dos diferentes fatores sobre o processo de aprendizagem é descrito por modelos matemáticos, propostos

5 The PBL learning process - www.pbli.org para essa finalidade, e é mensurado por meio da curva de aprendizagem.

Inicialmente introduzidas por Wright em 1936, as curvas de aprendizagem voltavam-se para a área de produção. Curva de aprendizagem é uma representação do nível médio cognitivo de aprendizagem para uma determinada atividade ou ferramenta. Normalmente, o aumento na retenção de informações é mais aguda após as tentativas iniciais e, então, gradualmente equilibra-se, o que significa que cada vez menos informação nova é retida após cada repetição ${ }^{6}$. Apresenta-se como uma ferramenta capaz de monitorar o desempenho não só de trabalhadores submetidos a tarefas repetitivas como também para analisar e programar tarefas, mostrando os entraves ou inabilidade do aprendiz. No presente trabalho, a curva de aprendizagem representa a dificuldade inicial de aprender algo e, em certa medida, o quanto há para aprender depois de familiaridade inicial. Sua utilização também permite a adequada alocação de atividades aos membros de um grupo, obedecendo suas características de atuação.

Dentre os vários modelos de curvas de aprendizagem, o mais usual é o modelo potencial. A base desse modelo consiste em desenvolver a ideia de uma curva de aprendizagem com foco no modelo de alimentação padrão:

$$
y(x)=\alpha x^{-\beta}
$$

onde $\boldsymbol{x}$ é a saída acumulada, medida em unidades, $y(x)$ é uma medida de custo médio ou o uso de entrada, e $\alpha$ e $\beta$ são parâmetros. Uma restrição a essa forma funcional é que quando a saída $\boldsymbol{x}$ torna-se grande, $y(x)$ tende a zero. Então, se a medida de $\boldsymbol{y}$ no ciclo de análise for grande, o modelo potencial levará a zero unidades e, para a maioria dos novos processos tecnológicos submetidos a esse efeito de aprendizagem, o modelo (25) não é adequado. Conforme [10], o modelo de curva potencial é apto a ser utilizado em situações específicas de modelagem, assim como

6 Wikipedia:http://pt.wikipedia.org/wiki/CCurva\_de\_aprendizagem. 
em cenários onde se verifica esquecimento e nos quais é demandada a participação de máquinas. Ainda, esse modelo continua a ser a peça central em grande parte esforços pedagógicos, [11].

No tratamento de problemas complexos nas áreas das ciências exatas, tais como a matemática aplicada e a engenharia, é comum a utilização de uma ferramenta computacional que quase sempre produz duas emoções por parte do utilizador, o sucesso e o fracasso. Busca-se ultrapassar a frustração de falhar em um processo. Ao grau de sucesso absorvido e obtido por cada um de nós durante o tempo, no processo de aprendizagem, denominamos curva de aprendizagem. Uma curva de aprendizagem mais lenta normalmente enfoca o lado experimental. Uma curva de aprendizagem mais rápida sustenta-se em potencialidades didáticas mais eficientes.

Dentre os fatores que afetam a curva de aprendizagem no ambiente de simulação de problemas no ensino de administração financeira, podemos citar:

- habilidade do acadêmico no trato dos temas em administração;

- $\quad$ bagagem de conhecimento em gestão financeira;

- $\quad$ treinamento em caixa preta (box training);

- $\quad$ treinamento em uso de ferramentas computacionais;

- $\quad$ acesso a esquemas estruturados de treinamento.

\section{Resultados}

A pesquisa foi conduzida durante o curso de extensão em modelagem computacional aplicada a problemas em administração. Os alunos foram motivados a participar preenchendo relatórios sobre os tempos necessários para atingir cada uma das etapas: leitura dos conceitos básicos, montagem do modelo matemático e aplicação a um caso de uma empresa fictícia selecionada em um conjunto de empresas com mesmas características. Um grupo desenvolveu os processos sem utilizar uma ferramenta computacional específica para a solução do problema. Um outro grupo desenvolveu os processos com aprendizagem assistida por computador (software Mathematica).

A aprendizagem foi acompanhada por duplas de monitores: um deles com habilidade no uso do software e o outro no modelo de gestão financeira. Além de orientar as fases da aprendizagem, os monitores também fizeram a verificação do tempo empregado pelos alunos e preenchimento dos relatórios de tempos. A partir dos relatórios obteve-se um parâmetro de ajuste da curva teórica (aprendizagem sem assistência computacional) e foi construída uma curva média para a aprendizagem assistida por computador.

No presente estudo, os valores outliers foram desconsiderados de forma a excluir fatores que melhorariam o desempenho dos resultados.

\section{Conclusão}

Este estudo mostrou que um ambiente simulado de aprendizado assistido por computador possibilita focalizar a atenção do aluno no problema e permite uma interpretação mais ágil do problema com resultados que podem ser ampliados sem danos em qualidade e tempo de solução. Das avaliações dos acadêmicos e do processo de solução tecnológica, o ambiente simulado proporciona aprendizagem relacional, em que o aprendiz adquire determinadas habilidades, permitindo-lhe fazer relações com outros casos de análise de referência na área. Neste caso, a melhoria do processo de aprendizagem dá-se pela interação do aluno com o recurso tecnológico, tornando o processo de aprendizagem mais individual. Proporciona também a aprendizagem criativa, no qual o aluno faz associação ao desenvolvimento de novos esquemas mentais, possibilitando a interação entre pessoas e tecnologias e compartilhando objetivos comuns. Neste caso, a aprendizagem processa-se de forma mais participativa.

Quanto às curvas de aprendizagem, juntamente com a análise do efeito aprendizado propriamente dito, as curvas estimularam a busca por melhores formas de solução dos problemas. O próprio acompanhamento da duração do desenvolvimento das etapas fez com que as pessoas envolvidas no processo tivessem novas ideias de melhoria para a sua consecução através da escolha de novas variações do recurso tecnológico utilizado para uma certa etapa. A documentação dos processos acabou sendo uma forma de implementação de melhorias no uso do software para a transferência de possibilidades de solução do problema.

Um dos cuidados que deve ser tomado na coleta de dados para os gráficos das curvas é a observação sem falhas para garantir que as informações sejam reais. Uma proposta de continuidade deste trabalho é avaliar outros modelos de curvas de aprendizagem aplicados ao problema de gestão financeira e adoção de técnicas estatísticas que melhorem a confiabilidade das informações obtidas.

\section{REFERENCES}

[1] G. J. R. Parada. "Margen-Rotación: un enfoque analítico," Economia y Administración, vol 32, pp. 91-123, 1987.

[2] M. P. Pinto. Uma abordagem de aprendizado assistido por computador utilizando programação simbólico-numérica, Ciência da Computação-UFC, Master Thesis, 1990.

[3] L. R. M. Teixeira. "A análise de erros: uma perspectiva cognitiva para compreender o processo de aprendizagem de conteúdos matemáticos," Nuances, vol III, pp. 47-52, setembro de 1997.

[4] R. Elizabetsky. Um modelo matemático para decisões de crédito no banco comercial., EPUSP-USP, Master Thesis, 1976.

[5] S. C. Kanitz. Como prever falências, McGraw Hill, 1978.

[6] J. R. Daza. "Insucesso de empresas," Revista Brasileira de Economia, vol 44, pp. 603-616, 1990.

[7] L. M. Onusic, S. P. de Castro Casa Nova, F. C. de Almeida. "Modelos de previsão de insolvência utilizando a análise por envoltória de dados: aplicação a empresas brasileiras," Revista de Administração Contemporânea, vol 11, online, 2007.

[8] S. Wolfran . The Mathematica Book, Cambridge University Press, 1999.

[9] T. H. Davenport, L. Prussak . Conhecimento Empresarial, Editora Campus, 1999.

[10] D. A. Nembhard, M. V. Uzumeri . "An Individual-Based Description of Learning within an Organization.," IEEE Transactions on Engineering Management, vol 47, pp. 370-378, 2000. http://dx.doi.org/10.1109/17.865905

[11] W. J. Hurley . "When are we going to change curve the learning lecture? ," Computers Operation Research, vol 23, pp 509-511, 1999. http://dx.doi.org/10.1016/0305-0548(95)00031-3

\section{AUTORES}

A. C. H. Marques é pesquisador da Universidade Federal de São Carlos, Rod. Washington Luis, Km 235 Caixa Postal 676, São Carlos - SP - Brasil (e-mail: achm@ufscar.br).

Submitted 19 March 2014. Published as re-submitted by the author 05 April 2014. 